\title{
A Numerical Analysis of Formation of the Surface Relief: a Single Inclusion Model
}

\author{
Olga Zinovieva ${ }^{1,2, a)}$, Varvara Romanova ${ }^{2,3, b)}$, Ruslan Balokhonov ${ }^{2,3, c),}$ \\ Valery Shakhijanov ${ }^{1, d)}$, and Tatiana Emelyanova, ${ }^{1, \mathrm{e}}$ \\ ${ }^{1}$ National Research Tomsk State University, Tomsk, 634050, Russia \\ ${ }^{2}$ Institute of Strength Physics and Materials Science SB RAS, Tomsk, 634055, Russia \\ ${ }^{3}$ National Research Tomsk Polytechnic University, Tomsk, 634050, Russia

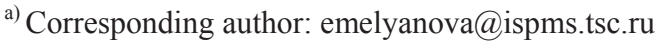 \\ b) varvara@ispms.tsc.ru \\ c) rusy@ispms.tsc.ru \\ d)arm3n1an@mail.ru \\ e) tv_em@mail.ru
}

\begin{abstract}
The influence of the material inhomogeneity on free surface roughening under uniaxial loading is simulated numerically in the framework of the mechanics of heterogeneous media, using a model of a single cubic inclusion embedded in a matrix as an example. Mechanical problems in 2D and 3D formulations are solved numerically by the finite-difference and finite-element methods. The stress-strain state responsible for the free surface roughening is examined. The effects of the inclusion orientation and location relative to the free surface and inclusion-to-matrix elastic modulus ratio on the surface relief characteristics are discussed.
\end{abstract}

Keywords: internal structure, strain-induced surface roughening, numerical simulation

\section{INTRODUCTION}

High-resolution experimental techniques used for scanning extended surface areas have revealed specific deformation phenomena developing on the free surface of the material. Strain-induced surface roughening occupies a special place here. On the one hand, surface roughening is an undesirable feature because it impairs the surface properties, such as reflectivity, lubricant transport and so on, and gives rise to plastic strain localization. On the other hand, the surface deformation pattern may be an indicator of the internal state of the material and can be used for nondestructive testing. Development of efficient methods to suppress or control surface roughening requires a deep understanding of the roughening mechanisms operative at different scale levels. While abundant experimental evidence has been produced and numerous theoretical studies have been performed, the problem of identifying the mechanisms involved and the factors responsible for the surface roughening in metals is the subject of considerable controversy.

In earlier papers (see, e.g., [1-3]), we have performed finite difference calculations with an explicit account of the material microstructure to investigate free surface roughening in uniaxial tension of polycrystalline and coated materials. Although the calculation results agree with the experimental evidence and provide a new insight into the roughening mechanisms, the amount of the information gained with the use of complex models is too vast and is nearly as difficult to analyze as the data obtained from real experiments. It is, therefore, a challenge to conduct simulations using simplified models to avoid the effect of the set of parameters used in the complex models. In this contribution, formation of the free surface relief in uniaxial tension is simulated numerically, using a single inclusion model. While a large number of papers have been devoted to analytical and numerical solutions of the mechanical problem on a single inclusion (see, e.g., $[4,5]$ ), free surface out-of-plane displacements have been excluded from consideration.

International Conference on Physical Mesomechanics of Multilevel Systems 2014

AIP Conf. Proc. 1623, 667-670 (2014); doi: 10.1063/1.4899033

(C) 2014 AIP Publishing LLC 978-0-7354-1260-6/\$30.00 


\section{MATERIAL MODELS AND LOADING CONDITIONS}

The 2D and 3D elastic problems of continuum mechanics are solved numerically by the finite-difference and finite-element methods [6,7]. Model specimens containing single cubic inclusions embedded in a matrix are presented in Fig. 1 $(\mathrm{a}-\mathrm{c})$. The inclusion orientation relative to the tensile axis was varied in the calculations. The geometrical models containing inclusions tilted at an angle of $22^{\circ}$ and $45^{\circ}$ to the tensile axis are shown in Fig. 1 $(\mathrm{a}-\mathrm{c})$. The location of the inclusion beneath the specimen upper surface was varied from 0 to a depth being equal to the linear size of the inclusion.

The elastic properties of the matrix match those of an aluminum alloy with the shear modulus $\mu_{\mathrm{m}}=27.7 \mathrm{GPa}$. The shear moduli of the inclusions $\mu_{\text {in }}$ are varied from 7 to $50 \mathrm{GPa}$ so that inclusion-to-matrix elastic modulus ratio varies between 0.25 and 1.75 .

The boundary conditions used in the 2D and 3D calculations (Fig. 1(a)) simulate uniaxial tension in the horizontal plane. The bottom surface is taken to be a symmetry plane, and the upper surface is free of external forces. A perfect mechanical contact at the matrix-inclusion interface is provided throughout the entire loading process. The 2D problem in terms of the plane strain is solved by the finite-difference method detailed in [6]. The 3D problem is solved using finite-element software ABAQUS.

\section{CALCULATION RESULTS AND DISCUSSION}

Figure $1(\mathrm{~d}-\mathrm{i})$ shows the $\sigma_{y y}$ stresses acting perpendicular to the free surface (hereinafter-the normal stresses) in specimens with inclusions oriented at different angles to the loading axis. In a homogeneous material, the normal stresses acting across the free surface are zero due to the absence of external forces on the surface. Thus, the free surface remains flat throughout the entire loading process. At the microscale level, however, where the material is strongly inhomogeneous, a complex stress-strain state arises near the internal interfaces, with all stress and strain tensor components being nonzero. The complex stress-strain state is exemplified by the calculations at hand.

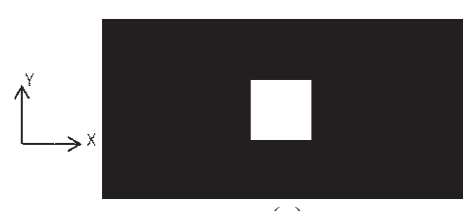

(a)

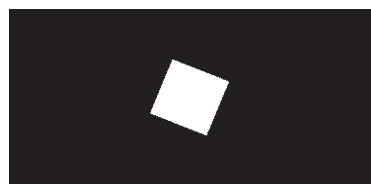

(b)

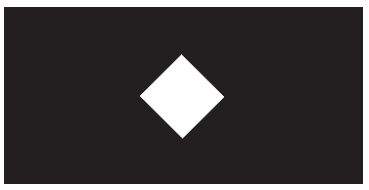

(c)

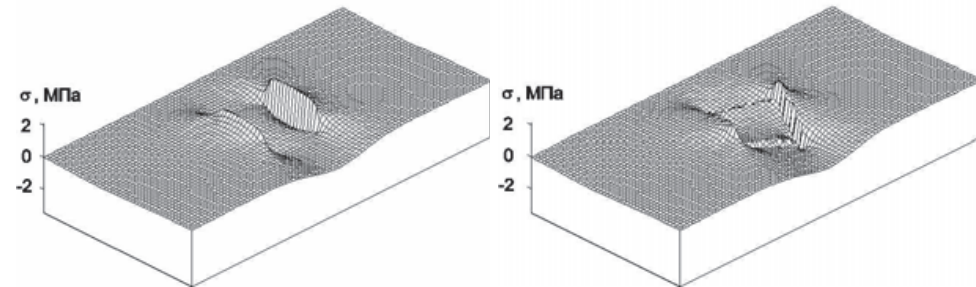

(d)

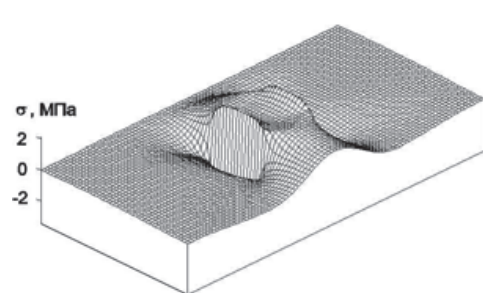

(g) (e)

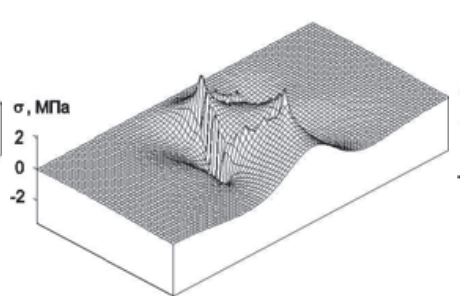

(h)

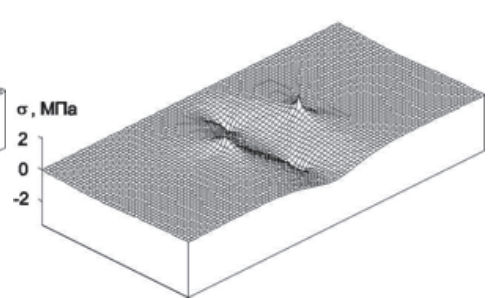

(f)

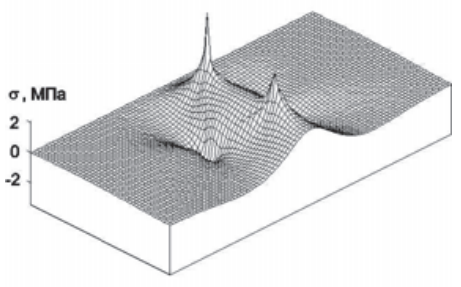

(i)

FIGURE 1. Two-dimensional models $(\mathrm{a}-\mathrm{c})$ and normal stress patterns for $\mu_{\mathrm{in}}>\mu_{\mathrm{m}}(\mathrm{d}-\mathrm{f})$ and $\mu_{\mathrm{in}}<\mu_{\mathrm{m}}(\mathrm{g}-\mathrm{i})$ 


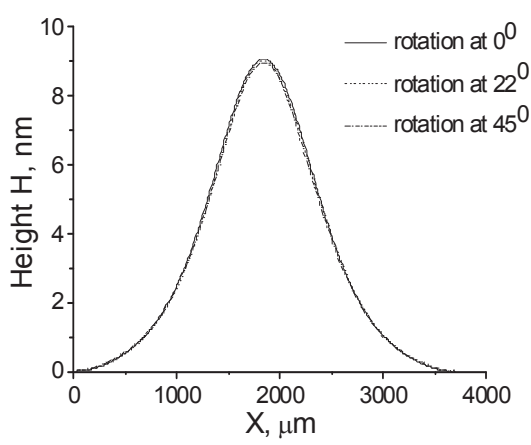

(a)

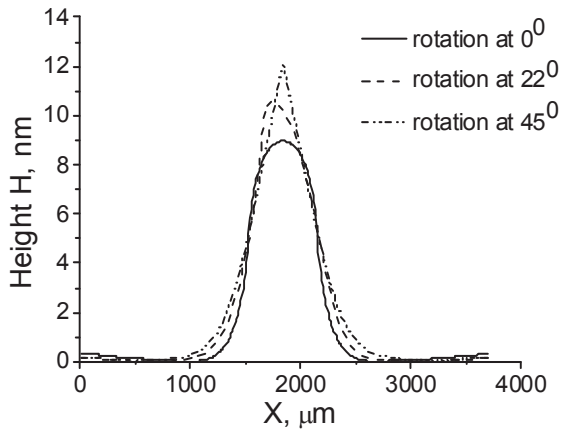

(b)

FIGURE 2. Surface profiles in specimens with hard inclusions located at a depth of 610 (a) and $0 \mu \mathrm{m}$ beneath the free surface (b) and oriented at different angles to the loading axis

The nonzero normal stresses $\sigma_{y y}$ occur at the matrix-inclusion interface with the positive and negative stress regions bordering each other (Fig. 1(d-i). Acting from the inside, the normal stresses induce inhomogeneous out-ofplane displacement fields on the free surface.

The calculation results presented in Figs. $1(\mathrm{~d}-\mathrm{f})$ and $1(\mathrm{~g}-\mathrm{i})$ were obtained for inclusions with higher and lower shear moduli with respect to the matrix. In what follows, the two cases will be referred to as a "hard" and a "soft" inclusion models. In the specimen with a hard inclusion, a hump is formed on the surface, whereas in the case of a soft inclusion, a dip is observed. The greater is the difference in the elastic properties between the matrix and the inclusion, the higher is the $\sigma_{y y}$ stress concentration at the matrix-inclusion interface.

The peak-to-valley distance of the surface folds is very small as compared with the linear size of the specimen. This is due to the fact that the normal stress level at the interfaces is low relative to the total stress level. However, experimental and theoretical studies (see, e.g., [8]) indicate that the surface roughness formed at an early loading stage grows almost linearly as plastic deformation develops.

Let us analyze the effect of the inclusion orientation relative to the direction of load application on the surface fold shape and height in terms of the mechanics of heterogeneous media. The calculations show that the inclusion orientation does not affect the fold shape and height when it is located at a distance being equal to the linear inclusion size beneath the free surface (Fig. 2(a)). When the inclusion is on the free surface, however, its orientation has a profound effect on the surface relief characteristics (Fig. 2(b)). In the case where the inclusion edges are parallel with the free surface plane and with the tensile axis, the surface fold is of the smallest height. When the inclusion is tilted at an angle of $45^{\circ}$, the fold demonstrates a sharp peak and a maximum height. When the inclusion is at an angle of $22^{\circ}$, the fold shape becomes asymmetric relative to the centerline.

Figure $3(\mathrm{a}, \mathrm{b})$ demonstrates a combined effect of the inclusion location and orientation. For the inclusion oriented at an angle of $45^{\circ}$ to the tensile axis, the peak-to-valley distance of surface folds exhibits a linear dependence on the inclusion location, whereas for the two other cases of the inclusion orientations, the dependence is nonlinear.

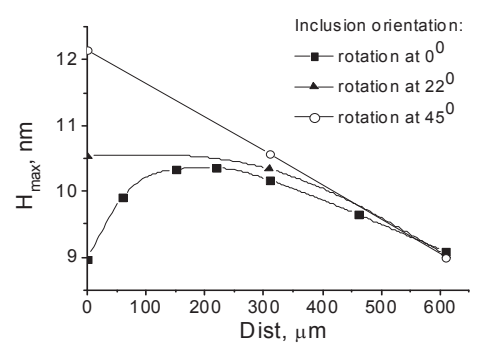

(a)

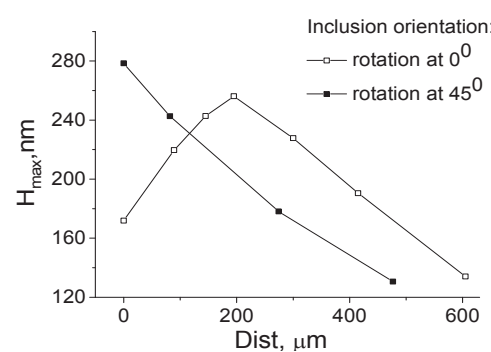

(b)

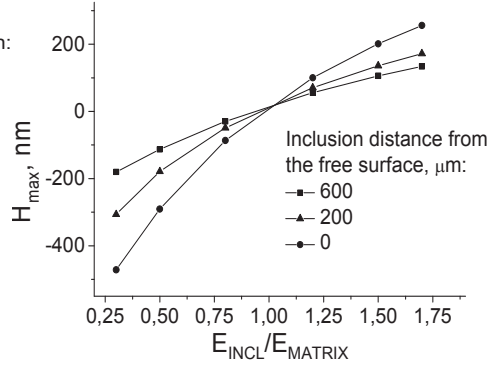

(c)

FIGURE 3. Peak-to-valley distance of surface folds vs. inclusion orientation, location and inclusion-to-matrix elastic modulus ratio: $2 \mathrm{D}$ calculations, $\varepsilon=0.01$ (a) and $3 \mathrm{D}$ calculations, $\varepsilon=0.5 \%(\mathrm{~b}-\mathrm{c})$ 


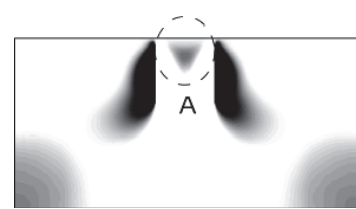

(a)

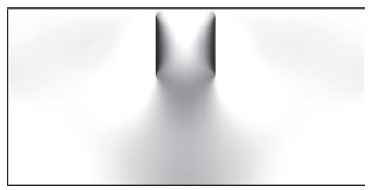

(e)

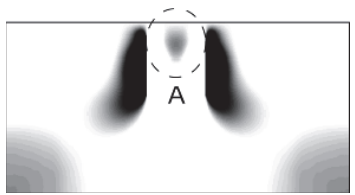

(b)

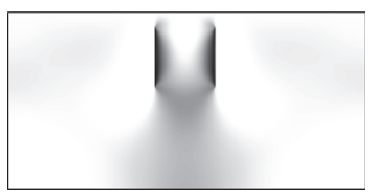

(f)

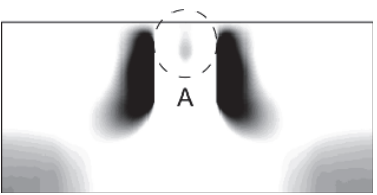

(c)

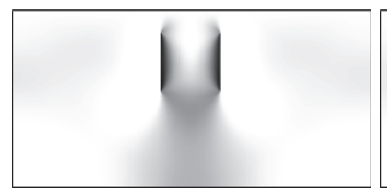

(g)

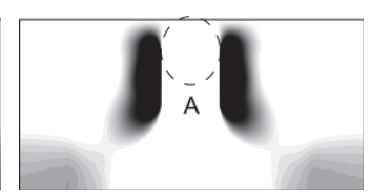

(d)

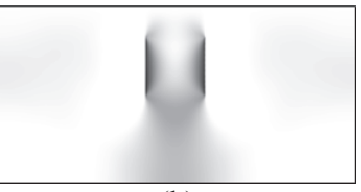

(h)

FIGURE 4. Tensile $(\mathrm{a}-\mathrm{d})$ and compressive stresses $\sigma_{y y}(\mathrm{e}-\mathrm{h}), \mu_{\mathrm{in}}>\mu_{\mathrm{m}}$

The non-linearity is most pronounced in the case of the inclusion whose edges are parallel with the pertinent specimen surfaces. Let us analyze the mechanical cause for the nonlinearity, using 2D calculations as an example (cf. Figs. 3(a) and 4). The maximum height of the surface folds is observed where the inclusion is located at a depth of $220 \mu \mathrm{m}$ below the free surface, which is about 0.4 of the inclusion size. This is due to the $\sigma_{y y}$ stress concentrations in the $A$ region (see Fig. 4). As the inclusion moves away from the free surface, the area of the $\sigma_{y y}$ tensile stresses becomes smaller and at a certain distance, the tensile stresses $\sigma_{y y}$ in the $A$ region demonstrate a compressive pattern. The smallest area of tensile stresses is observed where the inclusion is located at a depth of 0.4 of its linear size. As the tensile stress concentration in the $A$ region decreases, the surface fold height increases.

The combined effect of the inclusion location and inclusion-to-matrix elastic modulus ratio on the peak-to-valley distance is illustrated by the curves in Fig. 3(c). Obviously, the greater is the difference in the elastic properties between the matrix and the inclusion, the greater is the peak-to-valley distance, and the effect becomes more profound as the inclusion approaches the free surface.

\section{ACKNOWLEDGMENT}

The research was financed by a program to increase the competence of Tomsk Polytechnic University and by the Presidium of the Russian Academy of Sciences.

\section{REFERENCES}

1. V. A. Romanova, R. R. Balokhonov, and O. S. Emelyanova, Phys. Mesomech. 14(3), 159 (2011).

2. V. A. Romanova, R. R. Balokhonov, and S. Schmauder, Mater. Sci. Eng. A 564, 255 (2013).

3. V. A. Romanova and R. R. Balokhonov, Comput. Mater. Sci. 61, 71 (2012).

4. J. D. Eshelby, Proc. Roy. Soc. Lond. A. Math. Phys. Sci. 252(1271), 561 (1959).

5. T. Mori and K. Tanaka, Acta Metall. 21(5), 571 (1973).

6. M. Wilkins, Computer Simulation of Dynamic Phenomena (Springer-Verlag, Berlin, 1999).

7. V. P. Kuznetsov, I. Yu. Smolin, A. I. Dmitriev, D. A. Konovalov, A. V. Makarov, A. E. Kiryakov, and A. S. Yurovskikh, Phys. Mesomech. 16(1), 62 (2013).

8. M. R. Stoudt, J. B. Hubbard, and S. D. Leigh, Metall. Mater. Trans. A 42(9), 2668 (2011). 